\title{
Preliminary experiences of PET/MRI in predicting complete response in patients with breast cancer treated with neoadjuvant chemotherapy
}

\author{
CHIKAKO SEKINE ${ }^{1,2}$, NACHIKO UCHIYAMA $^{3}$, CHIKASHI WATASE $^{1}$, TAKESHI MURATA ${ }^{1}$, \\ SHO SHIINO $^{1}$, KENJIRO JIMBO ${ }^{1}$, ERIKO IWAMOTO ${ }^{1}$, SHIN TAKAYAMA ${ }^{1}$, HIROAKI KURIHARA ${ }^{3}$, \\ KAISHI SATOMI ${ }^{4}$, MASAYUKI YOSHIDA ${ }^{4}$, TAKAYUKI KINOSHITA ${ }^{5}$ and AKIHIKO SUTO ${ }^{1}$ \\ ${ }^{1}$ Department of Breast Surgery, National Cancer Center, Tokyo 104-0045; ${ }^{2}$ Department of Breast Surgery, \\ International University of Health and Welfare, Narita Hospital, Chiba 286-8520; \\ Departments of ${ }^{3}$ Radiology and ${ }^{4}$ Diagnostic Pathology, National Cancer Center, Tokyo 104-0045; \\ ${ }^{5}$ Department of Breast Surgery, National Hospital Organization Tokyo Medical Center, Tokyo 152-8902, Japan
}

Received September 13, 2021; Accepted December 7, 2021

DOI: $10.3892 / \mathrm{mco} .2021 .2483$

\begin{abstract}
Clinical response predictions through image examinations after neoadjuvant chemotherapy (NAC) for breast cancer is important. The present study aimed to evaluate the utility of a novel imaging modality, positron-emission tomography/magnetic resonance imaging (PET/MRI), in predicting the pathological complete response (pCR) to NAC in patients with early breast cancer. A total of 74 patients underwent PET/MRI, mammography (MG), including tomosynthesis, and ultrasound (US) after NAC. The complete response was predicted using each modality and these outcomes were compared accordingly. In terms of PET/MRI, complete response (CR) was defined as the disappearance of 18F-fluorodeoxyglucose uptake and the absence of enhanced lesions with contrast enhanced MRI. In MG and US, undetectable lesions were considered as CR. The background and tumor characteristics of patients were also analyzed between the pCR and non-pCR cases. Overall, 18 (24.3\%) of the 74 patients achieved $\mathrm{pCR}$. The overall sensitivity and
\end{abstract}

Correspondence to: $\mathrm{Dr}$ Chikako Sekine, Department of Breast Surgery, National Cancer Center, Tsukiji 5-1-1, Chuo-ku, Tokyo 104-0045, Japan

E-mail: chikako.s@jikei.ac.jp

Abbreviations: NAC, neoadjuvant chemotherapy; pCR, pathological complete response; MG, mammography; US, ultrasound; HER2, human epidermal growth factor receptor 2; FDG PET/CT, 18F-fluorodeoxyglucose positron-emission tomography/computed tomography; ER, estrogen receptor; PgR, progesterone receptor; FFDM, full-field digital mammography; DBT, digital breast tomosynthesis; CR, complete response

Key words: positron-emission tomography/magnetic resonance imaging, neoadjuvant chemotherapy, pathological complete response, early breast cancer specificity of PET/MRI were 72.2 and $78.6 \%$, respectively. Both the sensitivity in hormone receptor (HR)-positive cases and the specificity in HR-negative cases were $100 \%$. HR-negative and human epidermal growth factor receptor 2 (HER2)-positive cases demonstrated a significant association with $\mathrm{pCR}$ compared with HR-positive cases and triple negative cases $(\mathrm{P}=0.017)$. Furthermore, patients with 'mass' type lesions evaluated by MRI before NAC experienced pCR with a higher frequency than those with 'non-mass' type lesions. There was a statistically significant difference between the two groups ( $\mathrm{P}=0.018)$. In conclusion, PET/MRI is a different diagnostic approach that utilizes a multi-modality system. It demonstrates reasonable diagnostic accuracies of the responses of NAC with reference to hormonal subtypes in breast cancer.

\section{Introduction}

Neoadjuvant chemotherapy (NAC) is a well-established treatment for early breast cancer. NAC allows for breast conserving surgery by reducing the size of the tumor and provides an evaluation of real time sensitivity to therapy. This is very essential in determining the effectiveness of treatment. Preoperative assessment via imaging is important for surgical planning. It is also necessary to understand the characteristics of each imaging modality for diagnosis.

Dynamic contrast enhanced magnetic resonance imaging (MRI) has been widely used in breast cancer screening, determining the extent of disease, monitoring response to NAC, evaluating for rupture or cancer detection in patients with implants because of its high spatial resolution. ${ }^{18} \mathrm{~F}$-fluorodeoxyglucose positron-emission tomography/computed tomography (FDG PET/CT) has also been used in whole-body examination, assessing distant metastasis during initial staging and later surveillance. However, MRI with the breast extended in the supine position are more useful for diagnosing the condition inside the breast in detail.

In predicting pathological complete response ( $\mathrm{pCR}$ ) to NAC, MRI of the breast is more accurate compared with other 
imaging modalities, such as mammography (MG) and ultrasound (US) (1-5). FDG PET/CT is known to be an accurate imaging modality for response evaluation of NAC in breast cancer (6-8). Recently, PET/MRI, which can obtain images by combining metabolic analysis with PET and morphologic and vascularity analysis with contrast enhanced MRI simultaneously, has attracted attention as a new imaging modality. While several studies have reported the use of PET/MRI in breast cancer (9-11), the present study aimed to evaluate the efficacy of PET/MRI in the assessment of NAC.

\section{Materials and methods}

Patient selection and NAC regimen. This study protocol was approved by the local institutional review board, and written informed consent was obtained from all patients. Patients were not required to give informed consent to this study because the analysis used anonymous clinical data and images obtained after each patient agreed to treatment by written consent. We also applied the opt-out method to obtain consent for this study.

A total of 74 patients with stage II-III invasive breast cancer treated with NAC and surgery from September 2016 to March 2019 were enrolled, and the data were analyzed retrospectively. All patients underwent preoperative imaging evaluation with PET/MRI, MG, and US. Prior to NAC, 59 patients also underwent the same examinations before NAC while 15 patients underwent MRI, MG, and US. All the patients received anthracycline followed by a taxane regimen, except for two patients who received a platinum regimen followed by a taxane regimen. In addition, all patients with human epidermal growth factor receptor 2 (HER2) positive disease were treated with trastuzumab, and one of them was also treated with pertuzumab.

Imaging assessment through mammography and digital breast tomosynthesis. Clinical image data were acquired in the mediolateral-oblique and cranio-caudal positions using an a-Se full-field digital mammography (FFDM) system with a spatial resolution of $85 \mu \mathrm{m}$ (MAMMOMAT Inspiration, Siemens). In 63 patients (85.1\%), two-view digital breast tomosynthesis (DBT) was performed with the same compression angle and compression pressure as the FFDM. With one-view DBT, the radiation dose was 1.5 times of that with one-view FFDM. The radiation dose with ACR 156 was $1.2 \mathrm{mGy}$ with FFDM. FFDM and reconstructed $1 \mathrm{~mm}$ slice images from DBT were reviewed at a dedicated workstation. Complete response (CR) was defined as undetectable lesions or disappearance of density after NAC. Cases with only residual calcification were also defined as CR in this study.

Imaging assessment through ultrasound. Whole-breast US was performed with an $8 \mathrm{MHz}$ wide-band high-resolution transducer (aplio ${ }^{\mathrm{TM}} \mathrm{XV}$, Toshiba Medical Systems). The longest diameter of the hypoechoic part of the lesion was measured. Undetectable lesions were defined as CR.

Imaging assessment through PET/MRI. The images of PET/MRI that we evaluated in this study were organized from whole body PET/MRI images and breast PET/MRI images. Patients were instructed to fast for at least $4 \mathrm{~h}$ before the scan. Blood glucose levels were required to be $<350 \mathrm{mg} / \mathrm{dl}$.

Whole body PET/MRI. Whole body PET/MRI images were obtained using a PET scanner with 3T MRI (SIGNA; GE Healthcare). All PET images were acquired after intravenous injection of body weight-adapted ${ }^{18} \mathrm{~F}-\mathrm{FDG}$ doses $(4 \mathrm{MBq} / \mathrm{kg})$ followed by a resting period of 55-65 $\mathrm{min}$ in a supine position as the early phase. The data were recorded in 5-6 bed positions, for 2 min per bed position, and $2.8 \mathrm{~mm}$ imaging slices were obtained. The display field of view (DFOV) and matrix size were $50 \times 35 \mathrm{~cm}$ and $256 \times 256$, respectively. Images were reconstructed using the time-of-flight method (VUE Point) with four iterations and 32 subsets.

MR-based sequence for attenuation correction of PET images was performed using T1 weighted image (T1WI) with axial 3D-spoiled gradient echo (SPGR) sequence (LAVA-FLEX: FA/TR/TE: 5 degree/4 ms/1.7 ms, FOV: $50 \times 50 \mathrm{~cm}$, matrix size: $256 \times 128$, slice thickness: $5.2 \mathrm{~mm}$ ). Regarding whole body MRI, T1WI with axial 3D-SPGR sequence (LAVA: FA/TR/TE: 12 degree $/ 5400 \mathrm{~ms} / 2.6 \mathrm{~ms}$, FOV: $50 \times 37.5 \mathrm{~cm}$, matrix size: $512 \times 512$, slice thickness: $2 \mathrm{~mm}$ ) and T2 weighted image (T2WI) with single shot fast spin echo (SSFSE: FA/TR/TE: 90 degree/1600 ms/80 ms, FOV: $50 \times 37.5 \mathrm{~cm}$, matrix size: $512 \times 512$, slice thickness: $6 \mathrm{~mm}$ ) without fat suppression were obtained.

Breast PET/MRI. Late phase breast PET images were obtained 75-95 min after patients were given injections in a prone position. The DFOV and matrix size were $30 \mathrm{~cm}$ and 256x256. The images were reconstructed using the time-of-flight method (VUE Point) with four iterations and 32 subsets. The data were acquired for $10 \mathrm{~min}$ in a single bed position ( 89 slices, $249 \mathrm{~mm}$ ). Breast MRI was performed utilizing the 8-channel breast coil with fat suppression methods. The sequences consisted of axial T2WI (FSE-XL and IDEAL; FOV: 320x320, FA/TR/TE: 111 degree/5400 ms/80 ms, matrix size: $224 \times 320$, bandwidth: $90 \mathrm{kHz}$, slice thickness: $4 \mathrm{~mm}$ ), axial DWI (EPI and CHESS; FOV: 320x320, FA/TR/TE: 249 degree/6300 ms/67 ms, matrix size: 96x128, bandwidth: $250 \mathrm{kHz}$, slice thickness: $4 \mathrm{~mm}$ ). Dynamic contrast-enhanced axial T1WI consisted of pre-contrast and three post-contrast enhanced phases $(90,180$, and $270 \mathrm{sec}$ ) of T1WI (VIBRANT and CHESS; FOV: 320x320, FA/TR/TE: 12 degree $/ 5.3 \mathrm{~ms} / 2.4 \mathrm{~ms}$, matrix size: 400x400, bandwidth: $83 \mathrm{kHz}$, slice thickness: $3 \mathrm{~mm}$ ). Moreover, early contrast at $30 \mathrm{sec}$ after contrasted images (VIBRANT and CHESS; FOV: 320x320, FA/TR/TE: 12 degree $/ 5.3 \mathrm{~ms} / 2.1 \mathrm{~ms}$, matrix size: $224 \times 320$, bandwidth: $90 \mathrm{kHz}$, slice thickness: $3 \mathrm{~mm}$ ) were obtained. Meglumine gadoterate (Magnescope, Guerbet) was used as contrast agent, and it was automatically injected in the antecubital vein at $0.2 \mathrm{ml} / \mathrm{kg}$ bodyweight.

The breast PET and MR images were evaluated independently; the fusion images of early phase $(90 \mathrm{sec})$ dynamic contrast-enhanced T1WI and late phase PET were also assessed. The frequency of fusion of the images was organized from PET by $30 \%$ and MRI by $70 \%$. The data were analyzed by experienced radiologists.

Undetectable MRI enhancements without meaningful maximum standardized uptake values (SUVmax) of the tumor lesion of PET were defined as CR. Significant FDG uptake 
Table I. Patient characteristics.

\begin{tabular}{|c|c|c|c|c|c|c|}
\hline Patient characteristics & $\begin{array}{l}\text { Total } \\
(n=74)\end{array}$ & $\begin{array}{c}\mathrm{HR}^{+} / \mathrm{HER} 2^{-} \\
\quad(\mathrm{n}=30)\end{array}$ & $\begin{array}{c}\mathrm{HR}^{+} / \mathrm{HER} 2^{+} \\
\quad(\mathrm{n}=16)\end{array}$ & $\begin{array}{l}\mathrm{HR}^{-} / \mathrm{HER} 2^{+} \\
\quad(\mathrm{n}=10)\end{array}$ & $\begin{array}{l}\mathrm{HR}^{-} / \mathrm{HER} 2^{-} \\
(\mathrm{n}=18)\end{array}$ & P-value \\
\hline Age, years, median (range) & $48(30-78)$ & $44(30-78)$ & $50(37-69)$ & $58(45-71)$ & $50(33-78)$ & 0.14 \\
\hline Stage prior to NAC, n (\%) & & & & & & 0.11 \\
\hline 2 & $53(71.6)$ & $21(70.0)$ & $15(93.8)$ & $6(60.0)$ & $11(61.1)$ & \\
\hline 3 & $21(28.4)$ & $9(30.0)$ & $1(6.3)$ & $4(40.0)$ & $7(38.9)$ & \\
\hline Tumor-stage prior to NAC, n (\%) & & & & & & 0.58 \\
\hline $\mathrm{T} 1$ & $13(17.6)$ & $5(16.7)$ & $5(31.3)$ & $1(10.0)$ & $2(11.1)$ & \\
\hline $\mathrm{T} 2$ & $48(64.9)$ & $20(66.7)$ & $10(62.5)$ & $7(70.0)$ & $11(61.1)$ & \\
\hline $\mathrm{T} 3$ & $11(14.9)$ & $5(16.7)$ & $1(6.3)$ & $1(10.0)$ & $4(22.2)$ & \\
\hline $\mathrm{T} 4$ & $2(2.7)$ & $0(0.0)$ & $0(0.0)$ & $1(10.0)$ & $1(5.6)$ & \\
\hline Node-stage prior to NAC, n (\%) & & & & & & 0.61 \\
\hline No & $12(16.2)$ & $3(10.0)$ & $3(18.8)$ & $2(20.0)$ & $4(22.2)$ & \\
\hline N1 & $51(68.9)$ & $23(76.7)$ & $12(75.0)$ & $6(60.0)$ & $10(55.6)$ & \\
\hline $\mathrm{N} 2$ & $2(2.7)$ & $1(3.3)$ & $0(0.0)$ & $1(10.0)$ & $0(0.0)$ & \\
\hline N3 & $9(12.2)$ & $3(10.0)$ & $1(6.3)$ & $1(10.0)$ & $4(22.2)$ & \\
\hline Type of lesion on MRI, n (\%) & & & & & & 0.58 \\
\hline Mass & $27(36.5)$ & $9(30.0)$ & $7(43.8)$ & $3(30.0)$ & $8(44.4)$ & \\
\hline Non-mass & $40(54.1)$ & $19(63.3)$ & $9(56.3)$ & $5(50.0)$ & 7 (38.9) & \\
\hline NA & $7(9.5)$ & $2(6.7)$ & $0(0.0)$ & $2(20.0)$ & $3(16.7)$ & \\
\hline Histology, n (\%) & & & & & & 0.74 \\
\hline IDC & $71(95.9)$ & $28(93.3)$ & $16(100.0)$ & $9(90.0)$ & $18(100.0)$ & \\
\hline ILC & $1(1.4)$ & $1(3.3)$ & $0(0.0)$ & $0(0.0)$ & $0(0.0)$ & \\
\hline Metaplastic carcinoma & $2(2.7)$ & $1(3.3)$ & $0(0.0)$ & $1(10.0)$ & $0(0.0)$ & \\
\hline
\end{tabular}

HR, hormone receptor; HER2, human epidermal growth factor receptor 2; NAC neoadjuvant chemotherapy; MRI, magnetic resonance imaging; NA, not available; IDC, invasive ductal carcinoma; ILC, invasive lobular carcinoma.

(SUV values) was defined as those values that were higher than that for whole body average FDG uptake. The tumor shapes on early phase $(90 \mathrm{sec})$ dynamic contrast-enhanced MRI were classified into the 'mass type' and 'non-mass type'. The relationships between tumor shapes or molecular subtypes and pCR were also evaluated. The imaging data and diagnoses were analyzed by two experienced radiological specialists and breast surgeons with consensus.

Pathological assessment. Pathologic examination and immunohistochemistry were performed by dedicated breast pathologists. The histologic type of the tumor, tumor size, and histologic grade were determined from formalin-fixed paraffin-embedded tumor sections cut at a thickness of $4 \mu \mathrm{m}$ and stained with hematoxylin and eosin. The status of estrogen receptor (ER), progesterone receptor (PgR), and HER2 were analyzed with specimens of core needle biopsy before starting NAC. ER and PgR expressions were scored as positive or negative with a nuclear immunostaining cut-off of $1 \%$. ER and/or PgR positive tumors were defined as hormone receptor (HR) positive. HER2 was considered as positive if expression was scored at $3+$ in immunohistochemistry or if expression was scored at $2+$ with HER2 gene amplification by fluorescent in situ hybridization. pCR was defined as the absence of residual invasive cancer cells in the breast.
Statistical analysis. Cases with missing data were excluded. The characteristics among patients with invasive breast cancer were compared using Fisher's exact test. The sensitivity and specificity for the prediction of NAC effect were calculated for each modality. All data analyses were performed using Stata version 14 (Stata Corp.). All tests were two-sided, and P-values $<0.05$ were considered statistically significant.

\section{Results}

Patient and tumor characteristics. All patient and tumor characteristics are listed in Table I. A total of 74 women with stage II or III breast cancer were included in the study. Their mean age was 48 years (range, 30-78 years). Most of the tumors $(95.9 \%)$ were invasive ductal carcinomas. There was one case of invasive lobular carcinoma and two cases of metaplastic carcinomas. Four tumors were classified as histological grade 1 and 69 tumors as histological grade 2 or 3 . Immunohistochemical examination revealed that 30 patients

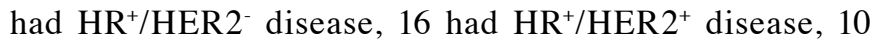
had HR-/HER $2^{+}$disease, and 18 had HR-/HER2- disease. According to the MRIs obtained before NAC, 27 (36.5\%) of the tumors were 'mass' type lesions and 40 (54.1\%) were 'non-mass' type lesions. 
Table II. Comparison of patients who achieved pCR and non-pCR.

\begin{tabular}{|c|c|c|c|}
\hline Patient characteristics & $\mathrm{pCR}(\mathrm{n}=18)$ & Non-pCR $(n=56)$ & P-value \\
\hline Age, years, median (range) & $49(32-78)$ & $48(30-78)$ & 0.79 \\
\hline Stage, n (\%) & & & 0.76 \\
\hline 2 & $12(66.7)$ & $41(73.2)$ & \\
\hline 3 & $6(33.3)$ & $15(26.8)$ & \\
\hline Type of lesion, n (\%) & & & 0.02 \\
\hline Mass & $11(61.1)$ & $16(28.6)$ & \\
\hline Non-mass & $5(27.8)$ & $35(62.5)$ & \\
\hline NA & $2(11.1)$ & $5(8.9)$ & \\
\hline Histology, n (\%) & & & 0.57 \\
\hline IDC & $17(94.4)$ & $54(96.4)$ & \\
\hline ILC & $0(0.0)$ & $1(1.8)$ & \\
\hline Metaplastic carcinoma & $1(5.6)$ & $1(1.8)$ & \\
\hline Subtype, n (\%) & & & 0.02 \\
\hline $\mathrm{HR}^{+} / \mathrm{HER} 2^{-}$ & $3(16.7)$ & $27(48.2)$ & \\
\hline $\mathrm{HR}^{+} / \mathrm{HER} 2^{+}$ & $4(22.2)$ & $12(21.4)$ & \\
\hline $\mathrm{HR}^{-} / \mathrm{HER} 2^{+}$ & $6(33.3)$ & $4(7.1)$ & \\
\hline $\mathrm{HR}^{-} / \mathrm{HER} 2^{-}$ & $5(27.8)$ & $13(23.2)$ & \\
\hline
\end{tabular}

pCR, pathological complete response; HR, hormone receptor; HER2, human epidermal growth factor receptor 2; NA, not available; IDC, invasive ductal carcinoma; ILC, invasive lobular carcinoma.

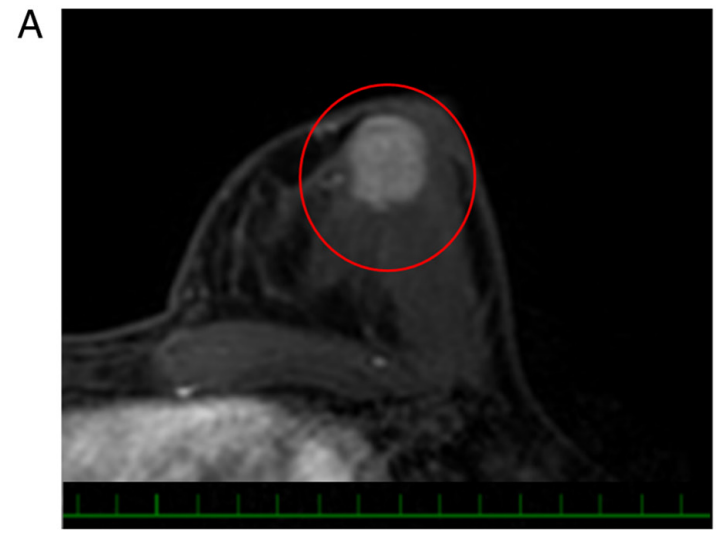

B

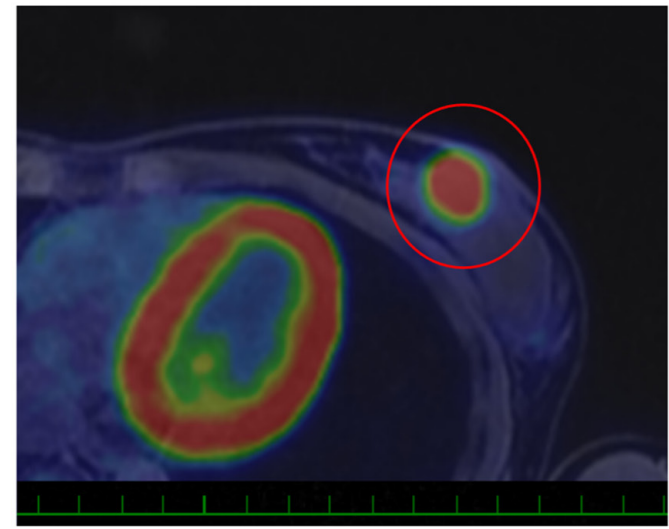

C

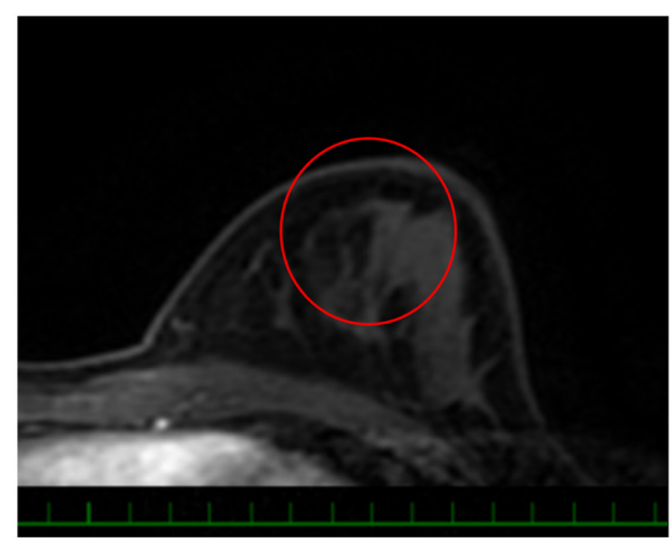

$\mathrm{D}$

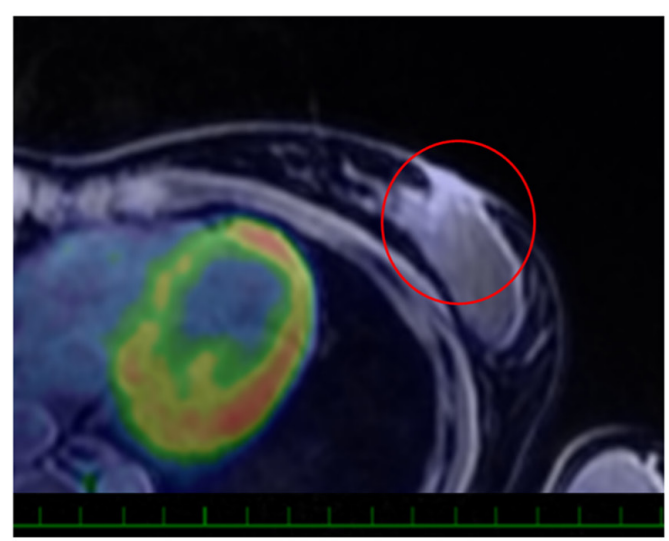

Figure 1. Typical imaging examples of a 'mass' type lesion of pCR case. A 32-year-old woman with hormone receptor-positive/human epidermal growth factor receptor 2-negative cancer (T2N1M0) on the lower inner quadrant of her left breast showed a good response to NAC. (A) Before NAC, the early phase of dynamic contrast-enhanced MRI with T1 weighted image (circle) showed an enhanced mass measuring 27 mm. (B) Fusion imaging of PET/MRI (circle) showed FDG uptake in the left breast with a measured SUVmax of 17.1. After NAC, (C) the enhanced mass disappeared (circle) and (D) the FDG uptake was not detected (circle). The tumor showed CR to NAC and the final pathological examination showed pCR. One scale of each scale bar, $1 \mathrm{~cm}$. pCR, pathological complete response; NAC, neoadjuvant chemotherapy; PET/MRI, positron-emission tomography/magnetic resonance imaging; FDG, 18F-fluorodeoxyglucose. 
A

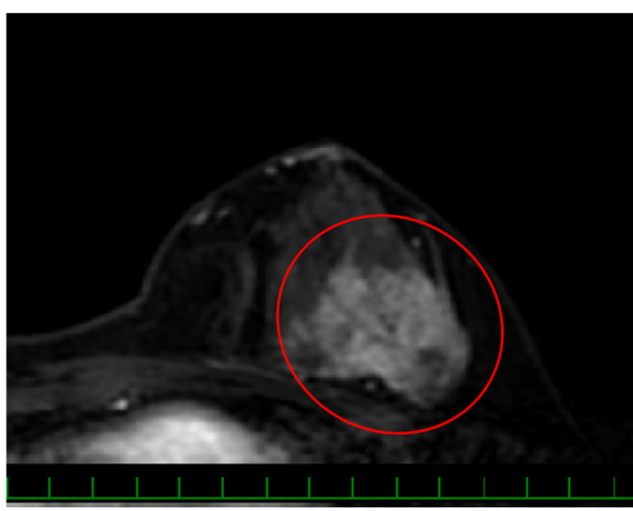

C

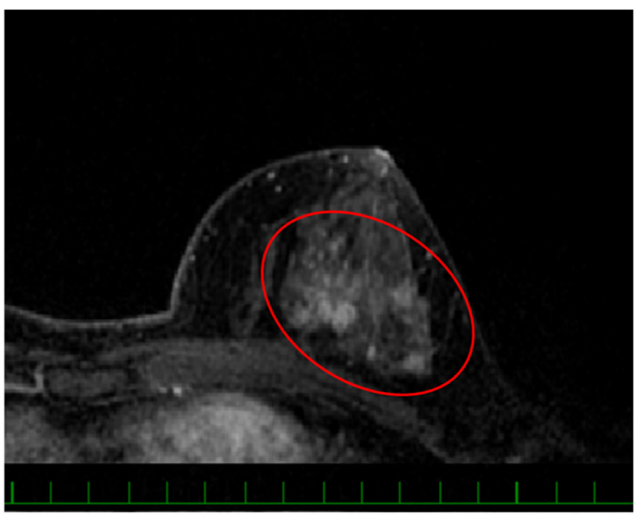

$\mathrm{B}$

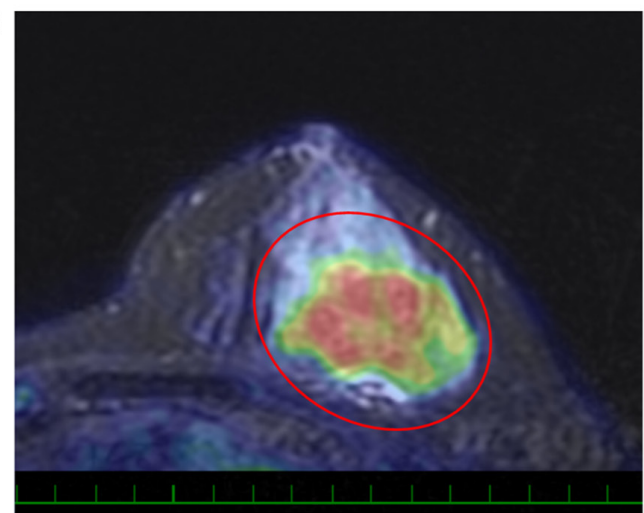

$\mathrm{D}$

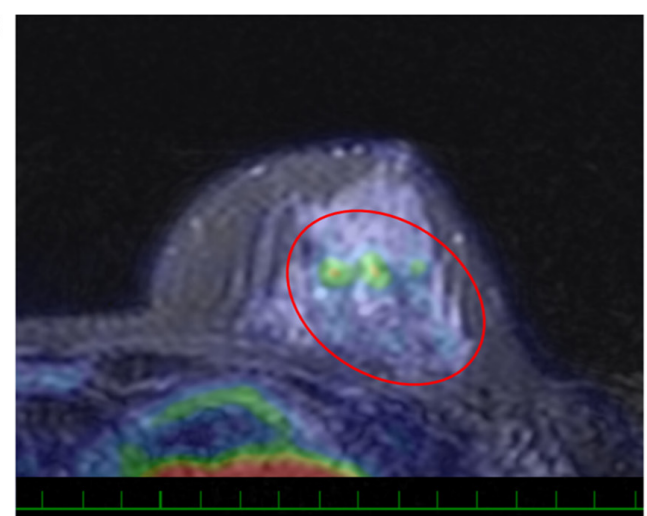

Figure 2. Typical imaging examples of a 'non-mass' type of non-pathological CR case. A 48-year-old woman with hormone receptor-positive/human epidermal growth factor receptor 2-negative cancer (T2N1M0) on the upper outer quadrant of her left breast showed partial response to NAC. (A) Before NAC, the early phase of dynamic contrast-enhanced MRI with T1 weighted image (circle) showed an irregular enhanced mass measuring $36 \mathrm{~mm}$. (B) Fusion imaging of PET/MRI (circle) showed an FDG uptake in the left breast with a measured SUVmax of 6.5. After NAC, (C) the enhanced mass showed a dendritic shrinkage pattern (circle) and (D) the FDG uptake was measured as 2.4 (circle). The tumor showed non-CR to NAC and the final pathological tumor size was $32 \mathrm{~mm}$. One scale of each scale bar, $1 \mathrm{~cm}$. CR, complete response; NAC, neoadjuvant chemotherapy; PET/MRI, positron-emission tomography/magnetic resonance imaging; FDG, 18F-fluorodeoxyglucose.

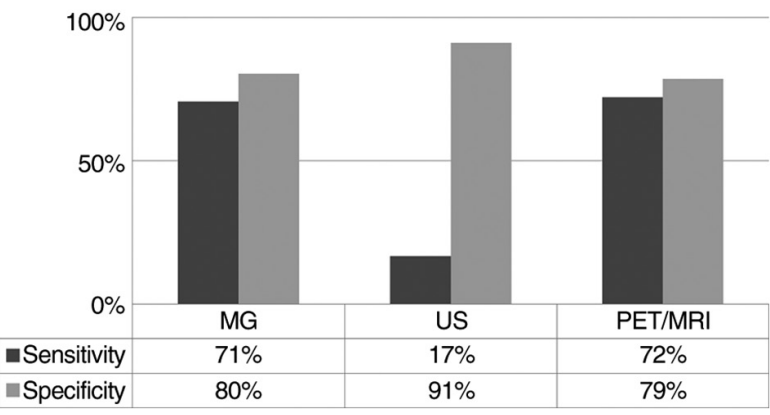

Figure 3. Sensitivity and specificity of the modalities in the entire patient cohort. MG, mammography; US, ultrasound; PET/MRI, positron-emission tomography/magnetic resonance imaging.

Identification by each modality. All the lesions were visually detectable using PET/MRI and US. Two lesions were difficult to identify using MG; these patients received only FFDM, and their data were excluded from the assessment of CR using MG.

$p C R$ rate and tumor characteristics. Eighteen patients (24.3\%) achieved pCR. HR negative and HER 2 positive cases demonstrated a significant correlation with $\mathrm{pCR}$ compared with HR positive cases and triple negative cases $(\mathrm{P}=0.017)$. Furthermore, patients with 'mass' type lesions who underwent MRI before NAC experienced pCR at a higher frequency than those with 'non-mass' type lesions (Table II). Typical responses to NAC are shown in Figs. 1 and 2.

$p C R$ prediction with PET/MRI. The overall sensitivity and specificity of pCR prediction with PET/MRI were 72.2 and $78.6 \%$, respectively. Among the 74 cases, 20 had undetectable enhancement on MRI and 51 cases did not demonstrate substantial SUV uptake on PET after NAC. Therefore, the accuracy of pCR prediction with PET/MRI depended more on the MRI than on the PET. Among pCR patients, 13 (72.2\%) showed undetectable enhancement of MRI and 17 patients (94.4\%) showed lack of meaningful SUVmax.

Sensitivity and specificity of $p C R$ prediction with each modality based on receptor status. The overall sensitivity and specificity of MG and US were 70.6 and $80.4 \%$, and 16.7 and $91.1 \%$, respectively (Fig. 3). In HR positive tumors, the sensitivity of PET/MRI was $100 \%$. In addition, in HR negative tumors, the specificity of PET/MRI was $100 \%$ (Fig. 4). None of the HR negative and HER2 positive patients were predicted to have achieved pCR with US.

\section{Discussion}

NAC is a standard therapy for locally advanced breast cancer as it allows tumor downstaging and facilitates breast 


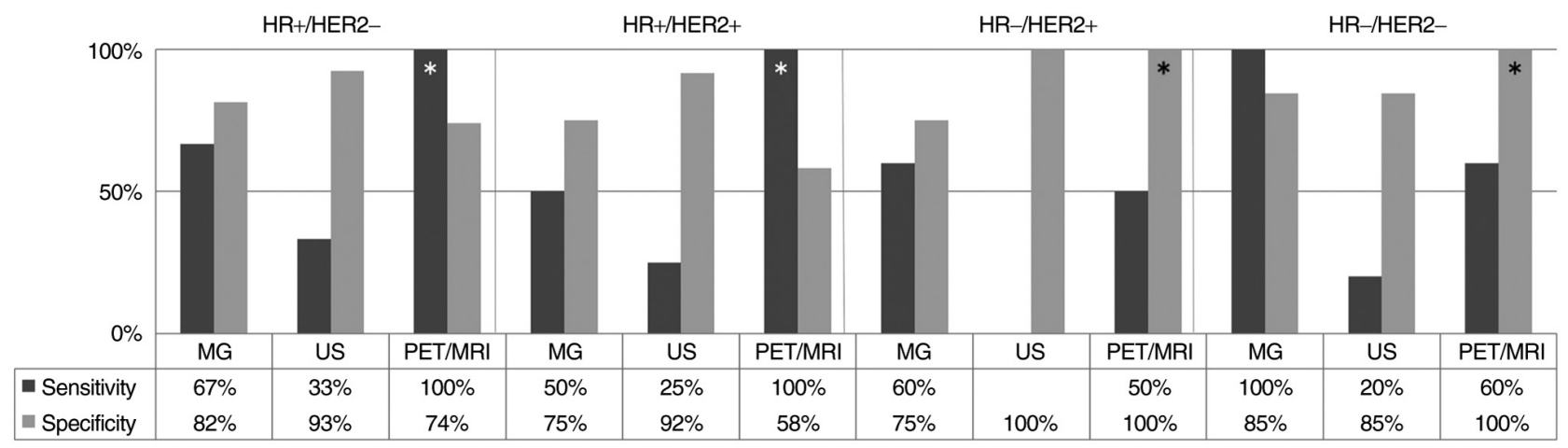

Figure 4. Sensitivity and specificity of pathological complete response prediction with each modality based on receptor status. *indicates the results of PET/MRI. HR, hormone receptor; HER2, human epidermal growth factor receptor 2; MG, mammography; US, ultrasound; PET/MRI, positron-emission tomography/magnetic resonance imaging.

conserving surgery. In addition, some trials have been reported wherein surgery was avoided in patients who achieved CR after NAC (12-14). Furthermore, the efficacy of six months of capecitabine administration after surgery for non-pCR patients has also been reported (15). At present, the accurate assessment of the effect of NAC is more important for the management of successful therapy than it was before.

MRI and PET/CT have been reported to be more accurate modalities for predicting pCR than both MG and US (16-19). The sensitivity and specificity of the $\mathrm{pCR}$ prediction were reported to be 65 and $88 \%$, respectively, for MRI, and 86 and $72 \%$, respectively, for PET/CT (20). PET/MRI is considered as a new modality that can make use of the advantages of both PET and MRI. In this study, the utility of PET/MRI for predicting pCR was assessed.

The overall sensitivity and specificity of PET/MRI were found to be acceptable. In particular, this study found that the sensitivity of PET/MRI in HR-positive tumors and the specificity in HR negative tumors were excellent; HER2 status did not affect the results. This meant that tumor disappearance was easily identified in HR positive tumors while the residual tumor was easily detected in HR negative tumors. This finding could be attributed to the association between the HR status and the morphological tumor features after NAC. HR positive tumors have an infiltrative shrinkage pattern and lower cellularity, whereas HR negative tumors are more likely to have a homogeneous tumor composition with centripetal shrinkage pattern and have significantly higher cancer cellularity than HR positive tumors (21).

Previous studies showed that the pCR prediction accuracy of MRI was higher in those with $H R$ negative tumors than in those with HR positive tumors $(22,23)$. Lee et al (21) explained this distinction as the difference in sensitivity to NAC. We also demonstrated that pCR was significantly more often observed among HR negative patients. Hence, the difference in prediction accuracy between this study and previous studies cannot be explained by the therapy effect alone. This difference may be attributed to improvements in PET and MRI accuracy or the technical difference between PET/MRI and PET/CT. Although former studies operated with a $1.5 \mathrm{~T}$ MRI systems, we used a 3.0T MRI system which has higher spatial resolution. While CT attenuation correction was based on the tissue density information provided by plain CT, MRI does not rely on tissue density. MRI contributes to soft-tissue contrast and vascularity information in detail. Dynamic contrast enhanced MRI allows measurement of the kinetic parameters related to permeability and perfusion. Moreover, advanced sequences, such as diffusion or apparent diffusion coefficient, which can provide helpful information, are available from MRI data. In addition, compared to $\mathrm{PET} / \mathrm{CT}$, PET/MRI in the prone position can be advantageous since clinicians can collect morphologic and metabolic imaging information simultaneously, and this might contribute to precision improvement.

When the response was analyzed according to the tumor shape on MRI, mass-type lesion was significantly related to pCR. Although HR-/HER2-tumors showed unifocal masses at the baseline more often (24), this cohort did not demonstrate obvious relationships between shapes on MRI and tumor subtypes. One explanation for this is provided by Loo et al (22), who showed that the earlier reported correlation between subtype and morphology of the tumor on MRI was valid for relatively large tumors selected for NAC (22).

During NAC, metabolic reduction within a tumor occurs much earlier than reduction in vascularity and shrinkage of tumor volume (25). Similarly, in our study, metabolic CR cases of PET were observed more frequently than cases with disappearance of enhancement on MRI. Metabolic analysis might investigate only the initial effect of NAC; therefore, by integrating it with morphology and vascularity analysis, a more accurate prediction may be possible.

In a previous assessment of each modality in $\mathrm{pCR}$ prediction, the sensitivity and specificity of MG were reported to be 48 and $81 \%$, respectively (26). The sensitivity of MG in the cohort of the current study was improved compared with that in this previous report. Although there are few studies on the efficacy of DBT in determining NAC effect, previous studies have demonstrated that DBT was also a useful modality like MRI $(3,27)$. Consistent with these findings, our result of pCR prediction with MG was appropriate because most of the tumors were evaluated via DBT. In addition, the pCR criteria for MG included residual microcalcification which explains the optimum results obtained. We considered that microcalcification without density on MG was from cancer treated previously, although residual calcification could be due to both treated cancer and a residual tumor. Therefore, DBT was more 
useful in detecting the density around areas of calcification compared to conventional MG.

In contrast, the sensitivity of US was insufficient. Croshaw et al (26) also reported low sensitivity (33\%) and high specificity $(90 \%)$ for US. We found that US could detect fibrosis and edema that was associated with complete response to NAC. When the US images of pCR patients diagnosed as non-pCR were reviewed, all of the detected lesions were described as hypoechoic areas and did not form mass shapes. With careful observation using US, the specificity was extremely good.

Although our study identified the usefulness of PET/MRI in the prediction of response to NAC, it has several limitations. First, we defined pCR as having no residual invasive disease. Consequently, our results might not be relevant to cases that do not include surgery as an option for patients with expected pCR. Despite this, there is no evidence that residual in situ carcinoma increases future distant relapse risk (28-31). Second, this was a retrospective study with a small sample size. Third, most of patients were treated with anthracycline-based regimens. Therefore, our findings may not be applicable to patients who received other regimens. Further large trials should be performed to confirm the results of our study.

In conclusion, PET/MRI provides a different diagnostic approach consisting of a multi-modality system. Although the diagnostic accuracy of the responses to NAC was similar to previous imaging modalities, under specific conditions, the usefulness of PET/MRI was confirmed.

\section{Acknowledgements}

Not applicable.

\section{Funding}

No funding was received.

\section{Availability of data and materials}

The datasets used and/or analyzed during the current study are available from the corresponding author on reasonable request.

\section{Authors' contributions}

CS and NU conceived and designed the present study. CS wrote the manuscript with support from NU, HK, TK and AS. NU, HK, TK, CW, TM, SS, KJ, EI, ST and AS acquired imaging data. CW, TM, SS, KJ, EI, ST, TK and AS analyzed and interpreted the patient data regarding breast cancer and imaging features. KS and MY performed the histological examination of the breast cancer tissues. CS and NU confirm the authenticity of all the raw data. All authors read and approved the final manuscript.

\section{Ethics approval and consent to participate}

This study protocol was approved (approval no. 2017-278) by the local institutional review board of the National Cancer Center (Tokyo, Japan). Written informed consent was obtained from all patients.

\section{Patient consent for publication}

Not applicable.

\section{Competing interests}

The authors declare that they have no competing interests.

\section{References}

1. Balu-Maestro C, Chapellier C, Bleuse A, Chanalet I, Chauvel C and Largillier R: Imaging in evaluation of response to neoadjuvant breast cancer treatment benefits of MRI. Breast Cancer Res Treat 72: 145-152, 2002.

2. Drew PJ, Kerin MJ, Mahapatra T, Malone C, Monson JR, Turnbull LW and Fox JN: Evaluation of response to neoadjuvant chemoradiotherapy for locally advanced breast cancer with dynamic contrast-enhanced MRI of the breast. Eur J Surg Oncol 27: 617-620, 2001.

3. Shin HJ, Kim HH, Ahn JH, Kim SB, Jung KH, Gong G, Son BH and Ahn SH: Comparison of mammography, sonography, MRI and clinical examination in patients with locally advanced or inflammatory breast cancer who underwent neoadjuvant chemotherapy. Br J Radiol 84: 612-620, 2011.

4. Atkins JJ, Appleton CM, Fisher CS, Gao F and Margenthaler JA: Which imaging modality is superior for prediction of response to neoadjuvant chemotherapy in patients with triple negative breast cancer? J Oncol 2013: 964863, 2013.

5. Dialani D, Chadashxili T and Slanetz P: Role of imaging in neoadjuvant therapy for breast cancer. Ann Surg Oncol 22: 1416-1424, 2015.

6. Pengel KE, Koolen BB, Loo CE, Vogel WV, Wesseling J, Lips EH, Rutgers EJ, Valdés Olmos RA, Vrancken Peeters MJ, Rodenhuis S and Gilhuijs KG: Combined use of 18F-FDG $\mathrm{PET} / \mathrm{CT}$ and MRI for response monitoring of breast cancer during neoadjuvant chemotherapy. Eur J Nucl Med Mol Imaging 41: 1515-524, 2014.

7. Li H, Yao L, Jin P, Hu L, Li X, Guo T and Yang K: MRI and PET/CT for evaluation of the pathological response to neoadjuvant chemotherapy in breast cancer: A systematic review and meta-analysis. Breast 40: 106-115, 2018.

8. Pahk K, Kim S and Choe KG: Early prediction of pathological complete response in luminal B type neoadjuvant chemotherapy-treated breast cancer patients: Comparison between interim 18F-FDG PET/CT and MRI. Nucl Med Commun 36: 887-891, 2015.

9. Melsaether A and Moy L: Breast PET/MR imaging. Radiol Clin North Am 55: 579-589, 2017.

10. Rice SL and Friedman KP: Clinical PET-MR imaging in breast cancer and lung cancer. PET Clin 11: 387-402, 2016.

11. Catalano OA, Horn GL, Signore A, Iannace C, Lepore M, Vangel M, Luongo A, Catalano M, Lehman C, Salvatore M, et al: PET/MR in invasive ductal breast cancer: Correlation between imaging markers and histological phenotype. Br J Cancer 116: 893-902, 2017.

12. Heys SD and Chaturvedi S: Primary chemotherapy in breast cancer: The beginning of the end or the end of the beginning for the surgical oncologist? World J Surg Oncol 1: 14, 2003.

13. Rea D, Tomlins A and Francis A: Time to stop operating on breast cancer patients with pathological complete response? Eur J Surg Oncol 39: 924-930, 2013.

14. Mamounas EP: Impact of neoadjuvant chemotherapy on locoregional surgical treatment of breast cancer. Ann Surg Oncol 22: 1425-1433, 2015.

15. Masuda N, Lee SJ, Ohtani S, Im YH, Lee ES, Yokota I, Kuroi K, Im SA, Park BW, Kim SB, et al: Adjuvant capecitabine for breast cancer after preoperative chemotherapy. N Engl J Med 376: 2147-2159, 2017.

16. De Los Santos J, Bernreuter W, Keene K, Krontiras H, Carpenter J, Bland K, Cantor A and Forero A: Accuracy of breast magnetic resonance imaging in predicting pathologic response in patients treated with neoadjuvant chemotherapy. Clin Breast Cancer 11: 312-319, 2011.

17. Marinovich ML, Houssami N, Macaskill P, Sardanelli F, Irwig L, Mamounas EP, von Minckwitz G, Brennan ME and Ciatto S: Meta-analysis of magnetic resonance imaging in detecting residual breast cancer after neoadjuvant therapy. J Natl Cancer Inst 105: 321-333, 2013. 
18. Gu YL, Pan SM, Ren J, Yang ZX and Jiang GQ: Role of magnetic resonance imaging in detection of pathologic complete remission in breast cancer patients treated with neoadjuvant chemotherapy: A meta-analysis. Clin Breast Cancer 17: 245-255, 2017.

19. Akimoto E, Kadoya T, Kajitani K, Emi A, Shigematsu H, Ohara M, Masumoto N and Okada M: Role of ${ }^{18} \mathrm{~F}-\mathrm{PET} / \mathrm{CT}$ in predicting prognosis of patients with breast cancer after neoadjuvant chemotherapy. Clin Breast Cancer 1: 45-52, 2018.

20. Liu Q, Wang C, Li P, Liu J, Huang G and Song S: The role of (18)F-FDG PET/CT and MRI in assessing pathological complete response to neoadjuvant chemotherapy in patients with breast cancer: A systematic review and meta-analysis. Biomed Res Int 2016: 3746232, 2016.

21. Lee HJ, Song IH, Seo AN, Lim B, Kim JY, Lee JJ, Park IA, Shin J, Yu JH, Ahn JH and Gong G: Correlations between molecular subtypes and pathologic response patterns of breast cancers after neoadjuvant chemotherapy. Ann Sug Oncol 22: 392-400, 2015.

22. Loo CE, Straver ME, Rodenhuis S, Muller SH, Wesseling J, Vrancken Peeters MJ and Gilhuijs KG: Magnetic resonance imaging response monitoring of breast cancer during neoadjuvant chemotherapy: Relevance of breast cancer subtype. J Clin Oncol 29: 660-666, 2011.

23. Koolen BB, Pengel KE, Wesseling J, Vogel WV, Vrancken Peeters MJ, Vincent AD, Gilhuijs KG, Rodenhuis S, Rutgers EJ and Valdés Olmos RA: FDG PET/CT during neoadjuvant chemotherapy may predict response in ER-positive/HER2-negative and triple negative, but not in HER2-positive breast cancer. Breast 22: 691-697, 2013.

24. Tsunoda-Shimizu H, Hayashi N, Hamaoka T, Kawasaki T, Tsugawa K, Yagata H, Kikuchi M, Suzuki K and Nakamura S: Determining the morphological features of breast cancer and predicting the effects of neoadjuvant chemotherapy via diagnostic breast imaging. Breast Cancer 15: 133-140, 2008.

25. Kim TH, Yoon JK, Kang DK, Kang SY, Jung YS, Han S, Kim JY, Yim $\mathrm{H}$ and An YS: Value of volume-based metabolic parameters for predicting survival in breast cancer patients treated with neoadjuvant chemotherapy. Medicine (Baltimore) 95: e4605, 2016.
26. Croshaw R, Shapira-Wright H, Svensson E, Erb K and Julian T: Accuracy of clinical examination, digital mammogram, ultrasound, and MRI in determining postneoadjuvant pathologic tumor response in operable breast cancer patients. Ann Surg Oncol 18: 3160-3163, 2011.

27. Uchiyama N,Kinoshita T,Hojo T,Asaga S,Suzuki J,Kawawa Y and Otsuka K: Usefulness of adjunction of digital breast tomosynthesis (DBT) to full-field digital mammography (FFDM) in evaluation of pathological response after neoadjuvant chemotherapy (NAC) for breast cancer. Breast Imaging 7361: 354-361, 2012.

28. Jones RL, Lakhani SR, Ring AE, Ashley S, Walsh G and Smith IE: Pathological complete response and residual DCIS following neoadjuvant chemotherapy for breast carcinoma. Br J Cancer 94: 358-362, 2006.

29. Kaufmann M, Hortobagyi GN, Goldhirsch A, Scholl S, Makris A, Valagussa P, Blohmer JU, Eiermann W, Jackesz R, Jonat W, et al: Recommendations from an international expert panel on the use of neoadjuvant (primary) systemic treatment of operable breast cancer: An update. J Clin Oncol 24: 1940-1949, 2006.

30. Mazouni C, Peintinger F, Wan-Kau S, Andre F, Gonzalez-Angulo AM, Symmans WF, Meric-Bernstam F, Valero V, Hortobagyi GN and Pusztai L: Residual ductal carcinoma in situ in patients with complete eradication of invasive breast cancer after neoadjuvant chemotherapy does not adversely affect patient outcome. J Clin Oncol 25: 2650-2655, 2007.

31. Symmans WF, Peintinger F, Hatzis C, Rajan R, Kuerer H, Valero V, Assad L, Poniecka A, Hennessy B, Green M, et al: Measurement of residual breast cancer burden to predict survival after neoadjuvant chemotherapy. J Clin Oncol 25: 4414-4422, 2007.

(i) (2) This work is licensed under a Creative Commons Attribution-NonCommercial-NoDerivatives 4.0 International (CC BY-NC-ND 4.0) License. 